\title{
Frailty but not sarcopenia nor malnutrition increases the risk of developing COVID-19 in older community-dwelling adults
}

\author{
Laetitia Lengelé $^{1}$ (1) Médéa Locquet ${ }^{1} \cdot$ Michel Moutschen $^{2} \cdot$ Charlotte Beaudart $^{1,3} \cdot$ Jean-François Kaux ${ }^{3,4}$. \\ Sophie Gillain ${ }^{5} \cdot$ Jean-Yves Reginster ${ }^{1} \cdot$ Olivier Bruyère $^{1,3,4}$
}

Received: 16 July 2021 / Accepted: 25 September 2021 / Published online: 23 October 2021

(c) The Author(s), under exclusive licence to Springer Nature Switzerland AG 2021

\begin{abstract}
Background The identification of coronavirus disease 2019 (COVID-19) risk factors is requested to implement prevention strategies.

Aim To explore the associations between the COVID-19 incidence and malnutrition, sarcopenia, and frailty, identified as potential risk factors in previous cross-sectional studies.

Methods Malnutrition, sarcopenia, and frailty were assessed at the last available follow-up from the Sarcopenia and Physical Impairments with Advancing Age (SarcoPhAge) cohort (i.e., the fifth year that ended in 2019) according to the MiniNutritional Assessment short-form, the European Working Group on Sarcopenia in Older People (EWGSOP2), and the Fried criteria, respectively. Information regarding the COVID-19 was gathered by phone calls interviews in April 2021 to measure its self-declared incidence. Adjusted Cox regressions and Kaplan-Meier curves were performed.

Results The present study included 241 participants [median age 75.6 (73.0-80.6) years, 63.1\% women]. Among them, 27 participants (11.2\%) developed the non-fatal Covid-19. No significant increased risks of COVID-19 were observed in patients with malnutrition [adjusted HR 1.14 (0.26-5.07)] and sarcopenia [adjusted HR $1.25(0.35-4.42)$ ]. Nevertheless, the incidence of COVID-19 was significantly higher in frail (44.4\%) than in robust participants (8.5\%) [Adjusted HR 7.01 (2.69-18.25)], which was confirmed by the Kaplan-Meier curves $(p<0.001)$. Among the frailty syndrome components, a low physical activity level was the only one significantly associated with an increased risk of COVID-19 [adjusted HR 5.18 (1.37-19.54)]. Conclusion Despite some limitations in the methodology of this study (i.e., limited sample size, COVID-19 incidence selfreported and not assessed systematically using objective measurements) requiring careful consideration, an increased risk to develop COVID-19 was observed in the presence of the frailty syndrome. Further investigations are needed to elaborate on our findings.
\end{abstract}

Keywords Malnutrition $\cdot$ Sarcopenia $\cdot$ Frailty $\cdot$ COVID-19 $\cdot$ SarcoPhAge

Laetitia Lengelé

llengele@uliege.be

1 Division of Public Health, Epidemiology and Health Economics, WHO Collaborating Centre for Public Health Aspects of Musculoskeletal Health and Aging, University of Liège, CHU-Sart Tilman, Quartier Hôpital, Avenue Hippocrate 13 (Bât. B23), 4000 Liège, Belgium

2 Department of Infectious Diseases and General Internal Medicine, CHU Sart-Tilman, University of Liège, 4000 Liège, Belgium
3 Physical, Rehabilitation Medicine and Sports Traumatology, SportS2, University Hospital of Liège, 4000 Liège, Belgium

4 Department of Sport Rehabilitation Sciences, University of Liège, 4000 Liège, Belgium

5 Geriatrics Department, University Hospital of Liège, Liège, Belgium 


\section{Introduction}

In March 2020, the World Health Organization (WHO) declared coronavirus disease 2019 (COVID-19) a pandemic [1]. COVID-19 is caused by severe acute respiratory syndrome (SARS) coronavirus 2 (SARS-CoV-2), which was first identified in China in December 2019. The emergence of this novel infectious agent in the human population has had international repercussions on public health systems because of the worldwide unprecedented nature of the situation and, therefore, the lack of efficient and available preventive strategies and therapeutics, the exceptional general quarantine measures, and its urgency regarding the health, social, and economic impacts [2]. On May 15th, more than 160 million COVID-19 confirmed cases were counted, with more than 3 million declared deaths worldwide [3]. The clinical picture of this disease is multisystemic [4]. It manifests through respiratory syndromes (i.e., fever, dry cough, and fatigue), neurologic syndromes including skeletal muscle injury, and gastrointestinal symptoms (i.e., nausea, vomiting, and loose bowel movement) $[4,5]$. Its complexity lies in the fact that the symptoms may remain silent in some while becoming mild to severe in others [5]. The severity of the symptoms is defined by the host's immune response during the virus infection. When SARS-COV-2 contaminates the host, the immune system is activated and induces an acute inflammatory response by releasing many cytokines, chemokines, and lipid mediators to fight the pathogenic agent [5-7]. It is well documented that an uncontrolled inflammatory response leads to a more severe form of COVID-19, resulting in multi-organ failure and death [7].

Emerging evidence indicates that increasing age is associated with an elevated inflammatory response level, inflammaging, because of an impaired immune function called "immunosenescence" [8]. This low-grade chronic inflammation further exacerbates the vulnerability of the geriatric population to COVID-19 and its adverse, sometimes fatal, outcomes [9-12]. In addition, parallel to the patient's age, the decline in immune system capacity could be even higher in persons with pre-existing comorbidities, for example malnutrition, frailty, and sarcopenia. These three major geriatric conditions are well known to impact the immune system and enhance its dysfunction. Malnutrition can be defined as "a state resulting from a lack of intake or uptake of nutrition that leads to altered body composition and body cell mass leading to diminished physical and mental function and impaired clinical outcome from disease" [13]. Nutritional status is recognized as a fundamental determinant of health as it is related to inflammation [14] and has previously been related to higher infection susceptibility [9, 12, 14-18]. Frailty is a clinical state characterized by reduced physiological reserve affecting immune function and adaptation to acute stressors and is thus associated with impaired immunity response [19-21]. This ability is also impacted by sarcopenia, defined as a progressive decline in muscle mass and muscle strength [22], which is associated with reduced response capacities to metabolic stress and has also been identified as a risk factor for pneumonia in older adults $[23,24]$. For all these reasons and because they are highly prevalent among the geriatric population [25-27], malnutrition, sarcopenia, and frailty represent potential priority risk factors for COVID-19.

The study of potential risk factors that may increase susceptibility to developing COVID-19 is fundamental to implement targeted prevention strategies to decrease its health burden. Only two longitudinal studies have studied the impact of frailty status on the onset of COVID-19 indicating a non-significant relationship between the two conditions [28, 29] and none has investigated malnutrition or sarcopenia as risk factors. Therefore, based on their probable links with the impaired immune system and higher risk of infectious diseases, the objective of the present study was to explore whether malnutrition risk, frailty, and sarcopenia are risk factors for the incidence of COVID-19.

\section{Methods}

\section{Study population}

This study presents data from the follow-up of the Sarcopenia and Physical Impairments with Advancing Aged (SarcoPhAge) study, in which baseline data have been previously described [30]. In summary, community-dwelling older adult volunteers aged over 65 were recruited in 2013 from press advertisements and general, geriatric, osteoporosis, rehabilitation, and rheumatology departments from an outpatient clinic in Liège, Belgium. An amputated limb or a body mass index (BMI) above $50 \mathrm{~kg} / \mathrm{m}^{2}$ was the only exclusion criteria applied to meet the requirements for dual X-ray absorptiometry (DXA). The Ethics Committee of the Teaching Hospital of the University of Liege approved this study (reference 2012/277) with three amendments in 2015 and 2018 and the last amendment in December 2019. All participants gave their written informed consent. The present study was undertaken according to the Declaration of Helsinki, 1986, and in accordance with general data protection regulations (GDPR).

\section{Data collection}

The participants were assessed annually by a clinical research assistant who performed physical evaluations [e.g., 
handgrip strength and Short Physical Performance Battery (SPPB) test] and proposed health questionnaires (e.g., the EuroQol-5D [31], the SarQol questionnaire [32], and the short-form (SF) 36 physical component summary (PCS) score and the SF-36 mental health component summary (MCS) score [33]) in a university hospital. The last available follow-up evaluation for all subjects from the cohort was the fifth year (T5), which ended in June 2019 (Fig. S1).

\section{Nutritional status evaluation}

The risk of malnutrition was assessed using the short form of the Mini Nutritional Assessment (MNA-SF), which consists of six items [34]: involuntary weight loss, loss of appetite, mobility, psychological stress, neuropsychological problems (i.e., dementia), and low BMI. Based on the total score, the individuals were identified as well nourished ( $\geq 12$ points), at risk of malnutrition ( $8-11$ points), or malnourished $(<8$ points). Nevertheless, this variable was considered dichotomous in the present analysis. Therefore, the participants were classified as either well-nourished or, at least, at risk of malnutrition (including then those identified as malnourished). This nutritional screening tool is validated and relevant for older adults [35-37]. The short form was chosen for the present study instead of the full version of the MNA, as the former is faster for screening, more commonly used in clinical practice [38], and has a mean sensitivity of $87 \pm 10$ and a mean specificity of $85 \pm 15$ against the MNA full form [39].

\section{Sarcopenia}

The revised 2018 definition of the European Working Group on Sarcopenia in Older People (EWGSOP2), widely used internationally, has been applied to diagnose sarcopenia [22]. This definition requires the presence of low muscle strength and a low muscle mass, defined as follows:

1. Muscle strength $<27 \mathrm{~kg}$ for men and $<16 \mathrm{~kg}$ for women. Muscle strength was measured with a hand-held handgrip dynamometer (Saehan Corporation, MSD Europe Bvba, Brussels, Belgium) calibrated each year. In compliance with the Southampton protocol, the participants were asked to squeeze it as hard as they could three times per hand, and the highest value of the six measures was considered in our analyses [40].

2. Fat-free mass (i.e., total body mass except for the fat mass) index $<17 \mathrm{~kg} / \mathrm{m}^{2}$ in men and $<15 \mathrm{~kg} / \mathrm{m}^{2}$ in women or appendicular lean mass (i.e., the sum of the muscle mass in both arms and legs) index $<7 \mathrm{~kg} / \mathrm{m}^{2}$ in men and $<5.5 \mathrm{~kg} / \mathrm{m}^{2}$ in women. Whole-body DXA scans (Hologic Discovery A, USA), calibrated daily, were used to estimate these muscle parameters, which were then divided by height-squared to obtain their index values.

\section{Frailty status}

Frailty was diagnosed by the Fried definition, which comprises a total of five criteria [41]

1. Unintentional weight loss of more than $5 \%$ from the past year.

2. Low handgrip strength, measured with a hand-held dynamometer (Saehan Corporation, MSD Europe Bvba, Brussels, Belgium) calibrated each year that was below established thresholds based on sex and BMI.

3. Exhaustion, assessed as a self-reported measure, identified by two items from the Center for Epidemiological Studies' depression scale [42].

4. Low walking speed at a distance of $4.5 \mathrm{~m}$ that was below established thresholds based on sex and height.

5. Low physical activity level, a self-reported score of kilocalories expended per week based on the reported time in physical activity by the participants on the Minnesota scale [43] that was below established thresholds by sex.

The participants who meet a minimum of three components are considered frail, while those with deficits in one or two components are considered in a pre-frail stage, and those without any Fried criteria are identified as robust. For the purpose of the present study, frailty status was analyzed as a dichotomous variable, with participants being either frail or robust; the latter category included pre-frail and robust stages.

\section{Outcome measure}

The outcome measure was the incidence of COVID-19. For this objective, the participants from the SarcoPhAge cohort, or their physician when necessary (participants impossible to contact), were interviewed by phone calls in April 2021 to gather information related to COVID-19. Information regarding symptoms of COVID-19 and its severity was assessed using a semi-constructed interview developed by a panel of experts in infectious diseases and geriatric and public health. As COVID-19 emerged in Belgium in March 2020, we were able to measure its cumulative incidence between March 2020 and April 2021.

\section{Covariate data collection}

During the follow-up visits, socio-demographic and anamnestic data were also collected. Among these, the following variables were considered confounding factors for their potential impact on nutritional status, muscle health, and the 
frailty phenotype, based on the literature and previous studies in the SarcoPhAge cohort [30, 44-47]: age, sex, number of comorbidities per individual, number of drugs consumed per individual, and Mini-Mental State Examination (MMSE) scores [48].

\section{Statistical analysis}

The distribution of quantitative variables was evaluated by examining the difference between the mean and the median values, histograms, quantile-quantile plots, and Shapiro-Wilk tests. As they did not follow a Gaussian distribution, all the continuous variables are reported as the median ( 25 th percentile-75th percentile), and the binary variables were expressed as absolute $(\mathrm{N})$ and relative frequencies (\%).

Malnutrition risk, sarcopenia, and frailty were assessed at T5 (i.e., 2018-2019). The socio-demographic and anamnestic data of the participants at T5 were compared between participants who contracted COVID-19 or not during the follow-up period using the Mann-Whitney $U$ test or the Chi-square test for quantitative or binary variables, respectively. Then, the incidence of COVID-19 was measured and compared based on nutritional, sarcopenia, and frailty status using Chi-square tests or Fisher's exact tests when more appropriate. To evaluate the risk of contracting COVID-19 based on these potential risk factors, a Cox proportional hazards model was performed, providing a hazard ratio (HR) and its $95 \%$ confidence interval (95\% CI). First, a crude hazard ratio was measured, and second, an adjusted model that included the confounding variables mentioned above was performed. The adjusted models for malnutrition and sarcopenia also included self-reported physical activity level but not frailty to avoid overadjustment. For the risk factor(s) significantly associated with COVID-19, we then aimed to determine which components of the syndrome led to a greater risk of COVID-19 in crude and adjusted models. Finally, the Kaplan-Meier method was used to yield survival curves to estimate the impact of the potential risk factors on the incidence of COVID-19, and log-rank tests were performed to compare the survival curves among participants with or without these risk factors.

The SPSS Statistics 24 (IBM Corporation, Armonk, NY, USA) software package was used for the analyses. The results were considered statistically significant at the 5\% critical level.

\section{Results}

When the SarcoPhAge study was initiated in 2013-2014, the cohort comprised 534 older participants. From the total SarcoPhAge population, 275 were assessed at the fifth year of follow-up (i.e., between 2018 and 2019), and a total of 55 were lost to follow-up (10.3\%), 73 refused (13.7\%), 65 were not able to participate (12.2\%), and 66 died (12.4\%). Then, after a median time of 26 (23-29) months after their fifth year of follow-up, we were able to acquire information on the incidence of COVID-19 for 241 of them, because 12 were impossible to contact (4.4\%), 5 refused to participate (1.8\%), 3 did not have the cognitive capacities to answer (1.1\%), and 14 had died (5.1\%). Therefore, the present analyses were performed on a total sample of 241 participants (Fig. 1).
Fig. 1 Flowchart of the SarcoPhAge study

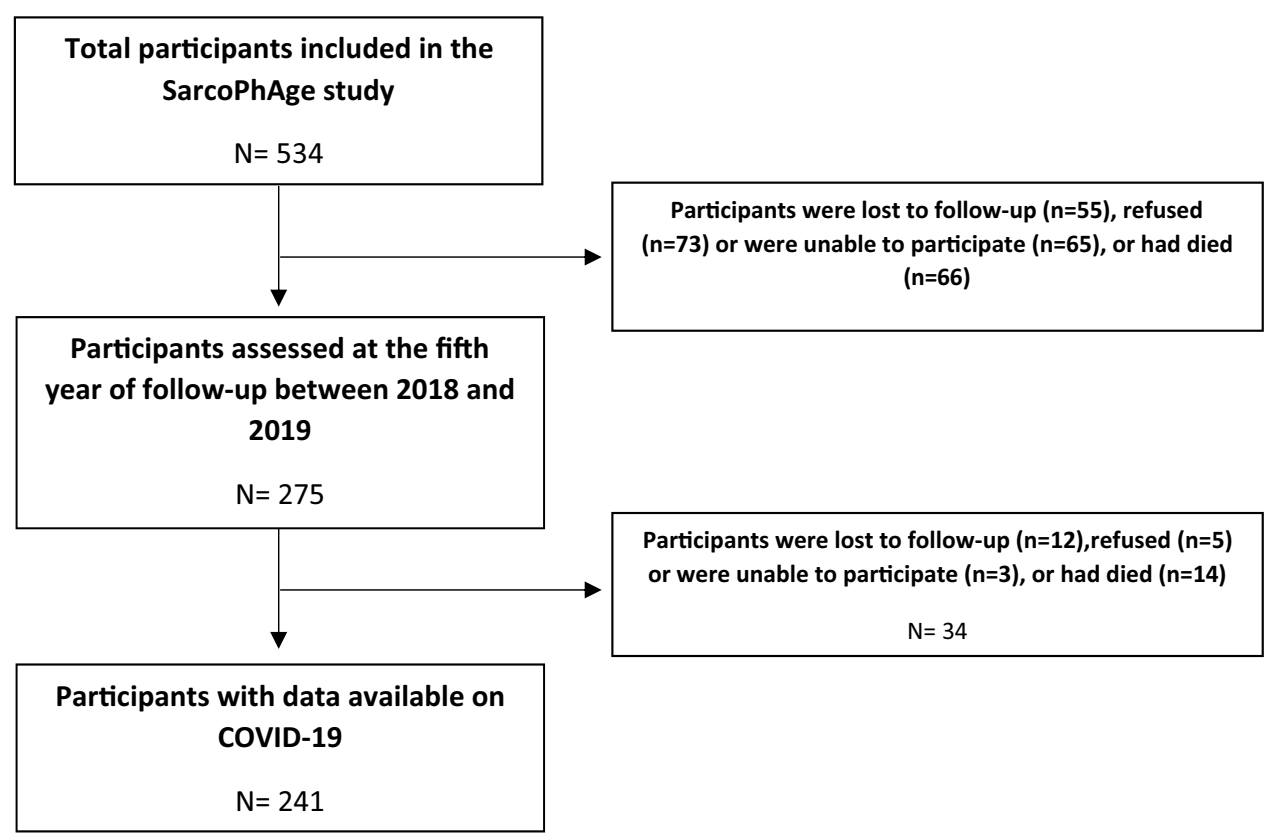


As expected, some of the baseline characteristics of the participants included in the present analyses were different from those of the nonparticipants. In particular, current participants, compared to the participants lost to follow-up, were at baseline significantly younger [69.8 (67.2-74.7) versus 75.2 (70.5-79.8) years], took fewer drugs per day [5.0 (3.0-7.0) versus $6.0(4.0-8.0)$ ], had fewer comorbidities [3.0 (2.0-5.0) versus $4.0(3.0-6.0)$ ], had a better cognitive status [29.0 (28.0-30.0) versus $28.0(27.0-29.0)]$, had a higher SPPB score [10.0 (9.0-11.0) versus 9.0 (7.0-11.0)], and a higher muscle strength $(28.0 \mathrm{~kg}(21.0-39.0 \mathrm{~kg})$ versus $25.0 \mathrm{~kg}(18.0-35.0 \mathrm{~kg}))$.

Table 1 displays the socio-demographic and health characteristics of the 241 participants included in the present analysis. The median age was $75.6(73.0-80.6)$ years, and $63.1 \%$ were women. Of them, 27 contracted COVID-19 during the follow-up (11.2\%). These participants did not significantly differ from those who did not contract the virus in terms of age, body mass index, number of drugs taken daily, number of comorbidities, cognitive status, level of physical activity, SPPB test score, muscle strength, and quality of life using both the EuroQol-5D and the SarQol questionnaire, with all $p$ values being above 0.05 .

Among the participants at risk of malnutrition at study inclusion, two (12.5\%) were diagnosed with COVID-19, and of those with sarcopenia, three (13.0\%) had COVID19 , compared to $25(11.1 \%)$ and $24(11.0 \%)$ well-nourished and non-sarcopenic participants, respectively (Table 2). The associations between the COVID-19 incidence and the risk of malnutrition resulted in a non-significant crude HR of 1.02 (0.24-4.29) and an HR of $1.14(0.26-5.07)$ in the adjusted model. Similar results were observed for sarcopenia, with a crude HR of $1.24(0.37-4.10)$ and an adjusted HR of 1.25 (0.35-4.42).

Out of the 18 participants identified as frail in the studied sample, 8 became positive for COVID-19 (44.4\%), compared to 19 in the robust group (8.5\%), revealing a crude HR of 5.84 (2.56-13.35). Indeed, the incidence of COVID-19 was significantly higher among the frail participants than among the robust participants, with a log-rank $p$ value $<0.001$ according to the Kaplan-Meier survival curve (Fig. 2). Regarding the number of symptoms and their severity (i.e., 8 have been hospitalized) among participants positive to COVID-19, there was no significant difference between groups according to the frailty status. After adjusting for confounding factors, an HR of 7.01 (2.69-18.25) was obtained, which indicated that the frail participants had a sevenfold higher risk of being affected by COVID-19.

As frailty status was significantly associated with a higher risk of developing COVID-19, we then aimed to determine which Fried diagnostic criteria led to a greater risk of COVID-19 (Table 3). Therefore, we analyzed the relationships between each criterion, namely, unintentional weight loss, low handgrip strength, exhaustion, low walking speed, and low physical activity level, and the incidence of COVID-19. Of all the Fried criteria, the COVID-19 incidence was significantly higher only in participants presenting exhaustion (20\%) or a low physical activity level (33.3\%) compared to those who did not meet these criteria (9.2\% and $10.6 \%$, respectively), with $p$ values of 0.04 for the exhaustion criterion and 0.03 for the physical activitylevel criterion. However, when we performed the survival analyses, the low physical activity-level criterion was the only criterion to remain significantly associated with a

Table 1 Sociodemographic and health characteristics of participants at inclusion in the study (T5)

\begin{tabular}{|c|c|c|c|c|}
\hline & \multirow{2}{*}{$\begin{array}{l}\text { Total studied sample } \\
(n=241)\end{array}$} & \multicolumn{3}{|c|}{ Incidence of COVID-19 } \\
\hline & & Yes $(n=27)$ & No $(n=214)$ & $p$ value \\
\hline Age, years & $75.6(73.0-80.6)$ & $75.3(72.3-78.5)$ & $75.6(73.1-80.7)$ & 0.38 \\
\hline Sex, women & $152(63.1)$ & $17(63.0)$ & $135(63.1)$ & 0.99 \\
\hline BMI, $\mathrm{kg} / \mathrm{m}^{2}$ & $26.5(23.6-29.7)$ & $27.4(24.0-29.7)$ & $26.4(23.6-29.7)$ & 0.41 \\
\hline Number of drugs per individual & $7.0(5.0-9.8)$ & $9.0(5.0-11.0)$ & $7.0(5.0-9.0)$ & 0.21 \\
\hline Number of comorbidities per individual & $4.0(3.0-5.0)$ & $4.0(3.0-7.0)$ & $4.0(2.0-5.0)$ & 0.16 \\
\hline Mini-Mental State Examination, /30 points & $28.0(27.0-29.0)$ & $29.0(27.0-29.0)$ & $28.0(27.0-29.0)$ & 0.85 \\
\hline Short Physical Performance Battery (SPPB), /12 points & $10.0(9.0-11.0)$ & $10.0(9.0-11.0)$ & $9.0(8.0-12.0)$ & 0.36 \\
\hline Muscle strength, kg & $19.0(14.0-19.0)$ & $19.0(14.5-29.0)$ & $18.0(12.0-28.0)$ & 0.16 \\
\hline \multicolumn{5}{|l|}{ Quality of life } \\
\hline EuroQol-5D ${ }^{\mathrm{a}}$ & $0.83(0.80-1.0)$ & $0.83(0.60-1.0)$ & $0.83(0.80-1.0)$ & 0.41 \\
\hline SF-36 PCS, $/ 100^{b}$ & 43.1 ( $34.9-51.0)$ & $40.5(26.6-50.3)$ & $43.1(35.5-51.5)$ & 0.16 \\
\hline SF-36 MCS, $/ 100^{b}$ & $46.9(36.6-53.7)$ & $48.3(40.3-54.8)$ & $46.7(36.4-53.6)$ & 0.48 \\
\hline SarQol $^{\mathrm{a}}$ & $64.0(51.5-75.2)$ & $65.1(41.6-72.9)$ & $63.8(52.7-75.7)$ & 0.73 \\
\hline
\end{tabular}

${ }^{a}$ Measured among 234 participants in total

${ }^{\mathrm{b}}$ Measured among 232 participants in total 
Table 2 Association between the risk of malnutrition (MNA-SF), sarcopenia (EWGSOP2), and frailty status (Fried criteria) and the incidence of COVID-19 $(n=241)$

\begin{tabular}{|c|c|c|c|c|c|}
\hline \multicolumn{6}{|c|}{ Risk of COVID-19 based on the malnutrition status } \\
\hline & \multicolumn{2}{|c|}{ Incidence of COVID-19 } & \multirow[t]{2}{*}{$p$ value } & \multirow{2}{*}{$\begin{array}{l}\text { Crude HR } \\
(95 \% \mathrm{CI})\end{array}$} & \multirow{2}{*}{$\begin{array}{l}\text { Adjusted }^{\text {a }} \\
\text { HR }(95 \% \text { CI })\end{array}$} \\
\hline & Yes $(n=27)$ & No $(n=214)$ & & & \\
\hline \multicolumn{6}{|c|}{ Malnutrition risk } \\
\hline Yes $(n=16)$ & $2(12.5)$ & $14(87.5)$ & \multirow[t]{2}{*}{0.70} & \multirow[t]{2}{*}{$1.02(0.24-4.29)$} & \multirow[t]{2}{*}{$1.14(0.26-5.07)$} \\
\hline No $(n=225)$ & $25(11.1)$ & $200(88.9)$ & & & \\
\hline \multicolumn{6}{|c|}{ Risk of COVID-19 based on the sarcopenia status } \\
\hline & \multicolumn{2}{|c|}{ Incidence of COVID-19 } & \multirow[t]{2}{*}{$p$ value } & \multirow{2}{*}{$\begin{array}{l}\text { Crude HR } \\
(95 \% \mathrm{CI})\end{array}$} & \multirow{2}{*}{$\begin{array}{l}\text { Adjusted }^{\text {b }} \\
\text { HR }(95 \% \text { CI })\end{array}$} \\
\hline & Yes $(n=27)$ & 214) & & & \\
\hline \multicolumn{6}{|l|}{ Sarcopenia } \\
\hline Yes $(n=23)$ & $3(13.0)$ & $20(87.0)$ & \multirow[t]{2}{*}{0.73} & \multirow[t]{2}{*}{$1.24(0.37-4.10)$} & \multirow[t]{2}{*}{$1.25(0.35-4.42)$} \\
\hline No $(n=218)$ & $24(11.0)$ & $194(89.0)$ & & & \\
\hline \multicolumn{6}{|c|}{ Risk of COVID-19 based on the frailty status } \\
\hline & \multicolumn{2}{|c|}{ Incidence of COVID-19 } & \multirow[t]{2}{*}{$p$ value } & \multirow{2}{*}{$\begin{array}{l}\text { Crude HR } \\
(95 \% \mathrm{CI})\end{array}$} & \multirow{2}{*}{$\begin{array}{l}\text { Adjusted }^{\mathrm{c}} \\
\text { HR }(95 \% \text { CI })\end{array}$} \\
\hline & Yes $(n=27)$ & $\overline{N o}(n=214)$ & & & \\
\hline \multicolumn{6}{|l|}{ Frailty } \\
\hline Yes $(n=18)$ & $8(44.4)$ & $10(55.6)$ & \multirow[t]{2}{*}{$<0.001$} & \multirow[t]{2}{*}{$5.84(2.56-13.35)$} & \multirow[t]{2}{*}{$7.01(2.69-18.25)$} \\
\hline No $(n=223)$ & $19(8.5)$ & $204(91.5)$ & & & \\
\hline
\end{tabular}

${ }^{a}$ Adjusted for age, sex, number of drugs per participant, number of comorbidities per participant, MMSE score, and level of physical activity (Minnesota)

${ }^{\mathrm{b}}$ Adjusted for age, sex, BMI, number of drugs per participant, number of comorbidities per participant, MMSE score, and level of physical activity (Minnesota)

${ }^{\mathrm{c}}$ Adjusted for age, sex, BMI, number of drugs per participant, number of comorbidities per participant, and MMSE score

higher risk of developing COVID-19 in the crude [HR of 4.15 (1.43-12.03)] and adjusted Cox models [HR of 5.18 (1.37-19.54)]. The participants with a low physical activity level had a fivefold higher risk of COVID-19 than the other participants.

\section{Discussion}

This study aimed to investigate the relationships between three major potential risk factors, namely, the risk of malnutrition, sarcopenia, and frailty, and the incidence of COVID-19 in community-dwelling older adults. A sevenfold increased risk of developing COVID-19 was observed in frail compared to robust participants in crude and adjusted models. However, frailty status was the only one out of the three geriatric diseases assessed in the present study to be significantly associated with a higher risk of COVID-19, as malnutrition and sarcopenia did not seem to predict its onset in the SarcoPhAge cohort. Moreover, of all the Fried criteria used to assess frailty, a low self-estimated physical activity level led to a fivefold greater risk of being affected by COVID-19 after adjusting for confounders.

To the best of our knowledge, no research has prospectively investigated the relationships between malnutrition or sarcopenia and the onset of COVID-19. Numerous crosssectional studies have observed a high prevalence of malnutrition among older patients hospitalized for COVID-19, with a pooled prevalence of $46.6 \%$ of them being malnourished [49]. Sarcopenia has been associated with conditions similar to some of the manifestations of COVID-19, such as pneumonia and acute respiratory distress syndrome [23, 24]. Although malnutrition and sarcopenia did not appear to be significant risk factors for the incidence of COVID-19 in the present study, the inverse relationship remains possible. Indeed, COVID-19 could be a risk factor for malnutrition due to its manifestation through gastrointestinal symptoms with nausea, vomiting, and loose bowel movement that could also cause anorexia [50]. In the same way, patients affected with COVID-19 have a decrease in their physical activity level because of isolation and fatigue, which can further accelerate the loss of muscle mass and strength and, therefore, engender sarcopenia [50, 51]. Additionally, given 


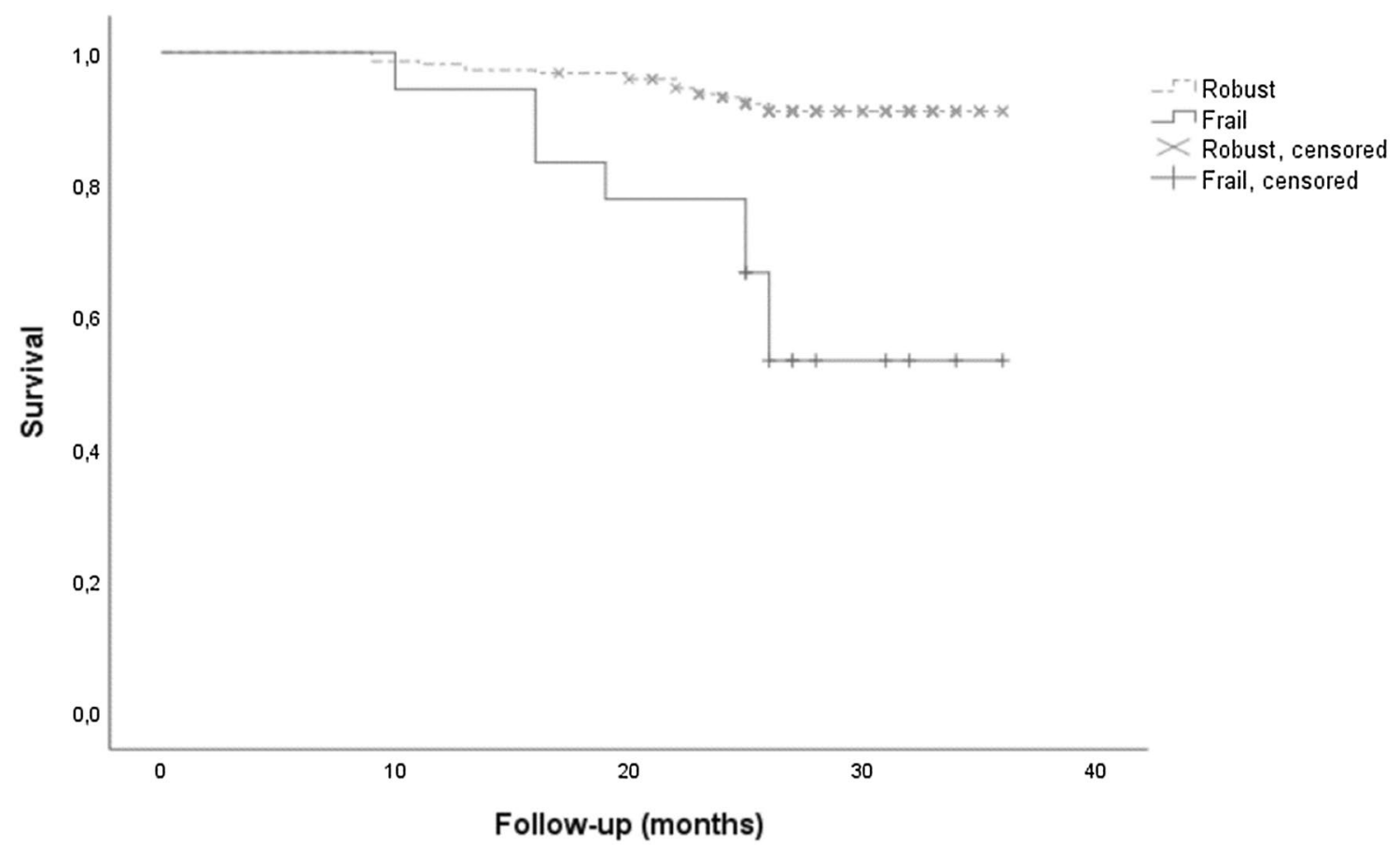

Fig. 2 Incidence of COVID-19 in robust or frail participants based on the Fried criteria

Table 3 Association between the different frailty criteria (Fried criteria) and the 2 year incidence of COVID-19 $(n=241)$

\begin{tabular}{|c|c|c|c|c|c|}
\hline \multicolumn{6}{|c|}{ Risk of COVID-19 based on each frailty criterion } \\
\hline & \multicolumn{2}{|c|}{ Incidence of COVID-19 } & \multirow[t]{2}{*}{$p$ value } & \multirow{2}{*}{$\begin{array}{l}\text { Crude HR } \\
(95 \% \mathrm{CI})\end{array}$} & \multirow{2}{*}{$\begin{array}{l}\text { Adjusted }^{\text {a }} \\
\text { HR }(95 \% \text { CI })\end{array}$} \\
\hline & Yes $(n=27)$ & No $(n=214)$ & & & \\
\hline \multicolumn{6}{|l|}{ Weight loss } \\
\hline Yes $(n=32)$ & $5(15.6)$ & $27(84.4)$ & 0.37 & $1.49(0.57-3.94)$ & $1.56(0.58-4.20)$ \\
\hline No $(n=209)$ & $22(10.5)$ & $187(89.5)$ & & & \\
\hline \multicolumn{6}{|c|}{ Low muscle strength } \\
\hline Yes $(n=146)$ & $17(11.6)$ & $129(88.4)$ & 0.79 & $1.16(0.53-2.53)$ & $1.18(0.50-2.76)$ \\
\hline No $(n=95)$ & $10(10.5)$ & 85 (89.5) & & & \\
\hline \multicolumn{6}{|l|}{ Exhaustion } \\
\hline Yes $(n=45)$ & $9(20.0)$ & $36(80.0)$ & 0.04 & $2.13(0.96-4.74)$ & $1.65(0.67-4.06)$ \\
\hline No $(n=196)$ & $18(9.2)$ & $178(90.8)$ & & & \\
\hline \multicolumn{6}{|c|}{ Low walking speed } \\
\hline Yes $(n=11)$ & $3(27.3)$ & $8(72.7)$ & 0.11 & $2.61(0.78-8.67)$ & $2.33(0.64-8.41)$ \\
\hline No $(n=230)$ & $24(10.4)$ & $206(89.6)$ & & & \\
\hline \multicolumn{6}{|c|}{ Low physical activity level } \\
\hline Yes $(n=12)$ & $4(33.3)$ & $8(66.7)$ & 0.03 & $4.15(1.43-12.03)$ & $5.18(1.37-19.54)$ \\
\hline No $(n=229)$ & $23(10.0)$ & $206(90.0)$ & & & \\
\hline
\end{tabular}

${ }^{a}$ Adjusted for age, sex, number of drugs per participant, number of comorbidities per participant, and MMSE score

their well-observed impact on the progression of COVID-19 with more extended hospitalization stays and a higher risk of mortality [52-55], these two clinical states still represent essential factors to be considered for better prevention and management of COVID-19 outcomes. Furthermore, as sarcopenia and malnutrition share overlapping factors in their assessment with the frailty phenotype, such as low muscle strength and unintentional weight loss, identify sarcopenic or malnourished individuals must be an integral part of the COVID-19 prevention. 
The association between frailty and the incidence of COVID-19 had previously been investigated by Woolford et al., whose results were contrary to ours, such that no relationship between the two conditions was observed [28]. In contrast to the present study, Woolford et al. included considerably more participants, approximately 4510, and COVID-19 was assessed through nasal and throat swab and respiratory secretion samples. However, several conditions in their study could explain our divergent results. First, their study population was mostly composed of adults aged between 40 and 69 years, compared to a geriatric population in our study, which is by definition at higher risk of developing the frailty phenotype and COVID-19. Second, the frailty assessment was made using a modified version [56] of the original definition reported by Fried and colleagues [41]. It can greatly impact the identification of frail participants, as different criteria measures and thresholds were used, specifically for exhaustion, slow walking speed, and low physical activity-level criteria. Finally, the diagnosis of the frailty phenotype was determined at the recruitment of the population between 2006 and 2010. The long period elapsing between the frailty status and COVID-19 incidence assessments implies that frail participants could have become robust during the follow-up, which is less likely to have happened in our study by assessing frailty status 2 years before the pandemic. A second population-based study in community-dwelling older adults in Ecuador also concluded that frailty status did not predispose them to SARS-CoV-2 infection [29]. Although COVID-19 has been diagnosed by SARS-CoV-2 antibody testing, the time of its diagnosis at the very beginning of the pandemic (i.e., May 2020) and the frailty criteria used, namely, the Edmonton frailty scale, are the two main reasons that make the comparison with our study challenging. The impact of the COVID-19 pandemic and its management is different from one country to another, which makes it even more challenging to compare studies conducted in different countries. Belgium is among the top five countries with the highest percentage of older adults above 80 years old [57], having thus a more at-risk population. Furthermore, the COVID-19 has brought an unprecedented crisis in the Belgian population because of excess mortality recorded between March and May 2020 [58]. Therefore, the Belgian health context and its impact on the health system may lead to a more challenging international comparison. Finally, the frailty phenotype has been significantly associated with higher mortality in COVID19-positive patients [59]. Alongside our results regarding the onset of COVID-19, it suggests that frailty impacts both the etiology and the severity of COVID- 19 .

The fact that frailty is significantly associated with the incidence of COVID-19, but not sarcopenia could be surprising, as these two conditions are well known to share common etiologies [60] and are both related to higher disability, morbidity, and mortality [61]. However, some assumptions regarding our results can be discussed. Even if sarcopenia and frailty have overlapping diagnostic criteria (i.e., low muscle strength), their operational definitions differentiate these two. Low muscle strength has to be associated with a low muscle mass to establish sarcopenia [22]. In contrast, frailty diagnosis requires low physical performance through low gait speed [41], which is a criterion considered for grading the severity of sarcopenia [22]. Furthermore, it is frequently observed that sarcopenia precedes frailty [61]. These hypotheses suggest that the frailty definition encompasses a more comprehensive assessment of fragility and disability in older people than sarcopenia, which was reflected in our analyses for the risk of COVID-19.

Among all the Fried components used to assess frailty in this study, a low physical activity level seems to be a major risk factor for the incidence of COVID-19. Its role in inflammation has already been evaluated in the previous studies with a focus on a process in which physical activity promotes an increase in muscle mass and a decrease in adipose tissue, triglycerides, and low-density lipoproteins, known to engender systemic inflammation and oxidative damage [62]. In one of the first studies on the subject in community-dwelling older adults, from Nilson et al., a significant impact on inflammation level was observed with physical activity of different intensity levels compared to sedentary condition [63]. Furthermore, evidence has suggested that regular exercise practices at moderate levels positively affected the immune system with increased immune surveillance and response [64]. Thus, physical activity can be highly beneficial in the context of respiratory infections such as COVID-19. Sedentary behavior is highly prevalent in the geriatric population, and the amount of time spent in physical activities decreases with advancing age [61, 65]. Nevertheless, sedentary behavior is a modifiable risk factor, and it has been shown that exercise interventions effectively prevent and even reverse the frailty process and are even more efficient than standard care alone [66]. Based on our results and the previous literature, physical activity and exercise interventions appear, therefore, to be relevant strategies that could be implemented in geriatric settings to counteract the age-associated decline in physical function and best prevent diseases that are more prevalent and severe among older adults, such as COVID-19. This also highlights the importance of COVID-19 vaccination in older adults, and especially in frail older adults as they are potentially at higher risk of COVID-19.

\section{Strengths and limitations}

The strength of the present study resides in its prospective design that is tailored for the analysis of risk factors. This study is the first to investigate malnutrition and sarcopenia 
as risk factors for COVID-19 and the first to use the Fried criteria for frailty. Moreover, sensibility analyses were performed on the Fried criteria with the weight loss threshold at more than $4.5 \mathrm{~kg}$ instead of a loss of 5\% and it assessed the robustness of our results regarding this risk factor (Table S1 in the supplementary materials). Indeed, among the 25 individuals identified as frail in the sensibility analyses, 8 became positive to COVID-19 (32.0\%) compared to 19 (8.8\%) in the robust group, leading to an adjusted HR of 3.97 (1.56-10.10). Moreover, when we analyzed the relationships between the $4.5 \mathrm{~kg}$ weight loss Fried criteria and the COVID-19 incidence, the same conclusions than with the $5 \%$ weight loss criteria were obtained with a non-significant adjusted HR of 0.91 (0.39-2.09) (Table S2). Therefore, the present study provides a better understanding of the pathogenesis of COVID-19. As new hypotheses were raised, this leads to new perspectives to be studied in future research to further improve understanding of the current health situation we are facing.

Nevertheless, this original study contains some limitations that need to be addressed. First, we assessed the risk of malnutrition using only the MNA-SF, but we did not diagnose malnutrition, as we did not have the required data (i.e., IGF-1) from the last available visit of the patients in the SarcoPhAge study to apply the GLIM criteria. However, this screening tool is considered relevant for the geriatric population [37]. Second, the analyses were performed on an already completed study cohort. Therefore, no sample size was calculated for statistical power prior to the present study, but we have realized post hoc analysis. It showed that we had indeed enough statistical power for the analyses regarding frailty (i.e., a minimum of 138 individuals required), while for malnutrition and sarcopenia, more patients were needed to demonstrate a significant difference for the incidence rate of COVID-19 within these groups. Therefore, caution is needed in interpreting the results regarding the low incidence of COVID-19 in the malnourished or sarcopenic participants. Furthermore, no deaths were reported in this high-risk population. Likely, the whole spectrum of the COVID-19 has not been covered in the present study. We can hypothesize that this can come from the 11 participants out of 27 positive to COVID-19 (40.7\%) who were already vaccinated at the time of outcome assessment. Indeed, the vaccinated participants are apparently more often completely asymptomatic, even if they are 65 years or older, as indicated by the study of Antonelli et al. [67]. Third, the physical activity level was self-reported. This measure is then susceptible to recall and social desirability bias and is thus prone to over reporting, but it was assessed using a validated questionnaire, namely, the Minnesota Leisure Time Physical Activity Questionnaire [68, 69]. The incidence of COVID-19 was also self-reported and not assessed by systematic tests such as blood sample tests. This suggests that some participants with light or no symptoms might not have been tested, and the incidence in our cohort might, therefore, be even higher than that observed in our analyses. Fourth, additional confounding factors could have been included in our analyses, especially the number of potential contacts with others and health-care providers. Indeed, the significant association between the frailty phenotype and higher incidence of COVID-19 could potentially be explained by the fact that frail individuals had more health-care contacts than non-frail ones, and that it exposed them to a higher risk of infection. However, this variable was not available in the SarcoPhAge cohort. Finally, our results could be limited because of an attrition bias impacting the estimated associations between the risk factors and COVID-19. Indeed, the participants included in the present study, compared to the participants lost to follow-up, were, at baseline, significantly younger, took fewer drugs per day, had fewer comorbidities, and had a better cognitive status. It can therefore be assumed that the participants of the present study were more robust than the general geriatric population.

\section{Conclusion}

An increased risk of COVID-19 was observed in the presence of frailty syndrome despite some limitations in the methodology of this study (i.e., limited sample size, COVID19 incidence self-reported and not assessed systematically using objective measurements) requiring careful consideration. Furthermore, out of all the frailty criteria, the participants with low physical activity had a significantly higher risk of experiencing COVID-19. The world is currently facing an unprecedented crisis due to the COVID-19 pandemic, and adapted prevention strategies are becoming essential to limit its health impact and burden. The present study results indicate that screening frailty status should be considered in the context of health policies to better target preventive actions. Moreover, malnutrition and sarcopenia could be necessary conditions to screen too concerning prevention of COVID-19 as these conditions share overlapping factors with frailty. Additionally, low physical activity seems to be a significant modifiable risk factor that has to be considered in the management of the COVID-19 crisis. Therefore, exercise intervention appears to be a relevant strategy to be implemented in geriatric settings. As we are the first to investigate these associations in a prospective cohort, further investigations are required to elaborate on our findings.

Supplementary Information The online version contains supplementary material available at https://doi.org/10.1007/s40520-021-01991-z.

Author contributions Conceptualization: ML, CB, J-YR, and OB; methodology: LL, ML, MM, CB, J-FK, and OB; formal analysis: LL; investigation: LL, and ML; writing —original draft preparation: LL; 
writing-review and editing: LL, ML, MM, CB, J-FK, SG, J-YR, and OB; resources: OB and J-YR; supervision: OB and J-YR. All authors have read and agreed to the published version of the manuscript.

Funding M.L. is supported by a fellowship from the FNRS (Fonds National de la Recherche Scientifque de Belgium-FRSFNRS—http:// www.frs-fnrs.be).

Data availability The datasets generated and/or analyzed in the current study are not publicly available due to GDPR policies and restrictions, because the information could compromise the privacy of the participants. However, the data are available from the corresponding author on reasonable request.

\section{Declarations}

Conflict of interest The authors declare no conflict of interest.

Ethical approval The guidelines of the Declaration of Helsinki were followed, and the present study was approved by the ethics committee of the University of Liege Teaching Hospital (reference 2012/277), with two amendments in 2015 and 2018.

Human and animal rights All procedures followed have been performed in accordance with the ethical standards laid down in the 1964 Declaration of Helsinki and its later amendments.

Consent to participate Informed consent was obtained from all participants involved in the study.

Consent for publication Informed consent was obtained from all participants involved in the study.

\section{References}

1. World Health Organization (WHO) (2020) WHO Director-General's opening remarks at the media briefing on COVID-19-11 March 2020

2. Chen LK (2020) Older adults and COVID-19 pandemic: resilience matters. Arch Gerontol Geriatr 89:104124. https://doi.org/ 10.1016/j.archger.2020.104124

3. Belgium: WHO Coronavirus Disease (COVID-19) Dashboard With Vaccination DatalWHO Coronavirus (COVID-19) Dashboard With Vaccination Data Available online: https://covid19. who.int/region/euro/country/be. Accessed on $10 \mathrm{Jul} 2021$

4. Willows S, Alam SB, Sandhu JK et al (2021) A Canadian perspective on severe acute respiratory syndrome coronavirus 2 infection and treatment: how prevalent underlying inflammatory disease contributes to pathogenesis. Biochem Cell Biol 99:173-194. https://doi.org/10.1139/bcb-2020-0341

5. Li G, Fan Y, Lai Y et al (2020) Coronavirus infections and immune responses. J Med Virol 92:424-432. https://doi.org/10. $1002 / \mathrm{jmv} .25685$

6. Zhang B, Zhou X, Qiu Y et al (2020) Clinical characteristics of 82 death cases with COVID-19. medRxiv. https://doi.org/10.1101/ 2020.02.26.20028191

7. Lingeswaran M, Goyal T, Ghosh R et al (2020) Inflammation, immunity and immunogenetics in COVID-19: a narrative review. Indian J Clin Biochem 35:260-273. https://doi.org/10.1007/ s12291-020-00897-3
8. Schoevaerdts D, Sibille FX, Gavazzi G (2021) Infections in the older population: what do we know? Aging Clin Exp Res 33:689_ 701. https://doi.org/10.1007/s40520-019-01375-4

9. Bencivenga L, Rengo G, Varricchi G (2020) Elderly at time of COronaVIrus disease 2019 (COVID-19): possible role of immunosenescence and malnutrition. Geroscience 42:1089-1092. https://doi.org/10.1007/s11357-020-00218-9

10. Salimi S, Hamlyn JM, Le Couteur D (2020) COVID-19 and crosstalk with the hallmarks of aging. J Gerontol 75:e34-e41. https://doi.org/10.1093/gerona/glaa149

11. Bajaj V, Gadi N, Spihlman AP et al (2021) Aging, immunity, and COVID-19: how age influences the host immune response to coronavirus infections? Front Physiol 11:1793. https://doi. org/10.3389/fphys.2020.571416

12. Wu C, Chen X, Cai Y et al (2020) Risk factors associated with acute respiratory distress syndrome and death in patients with coronavirus disease 2019 pneumonia in Wuhan, China. JAMA Intern Med 180:934-943. https://doi.org/10.1001/jamaintern med.2020.0994

13. Cederholm T, Barazzoni R, Austin P et al (2017) ESPEN guidelines on definitions and terminology of clinical nutrition. Clin Nutr 36:49-64. https://doi.org/10.1016/j.clnu.2016.09.004

14. De Araújo Morais AH, Aquino JDS, Da Silva-Maia JK et al (2021) Nutritional status, diet and viral respiratory infections: perspectives for severe acute respiratory syndrome coronavirus 2. Br J Nutr 125:851-862. https://doi.org/10.1017/S000711452 0003311

15. Suardi C, Cazzaniga E, Graci S et al (2021) Link between viral infections, immune system, inflammation and diet. Int J Environ Res Public Health 18:1-13. https://doi.org/10.3390/ijerph1805 2455

16. Barazzoni R, Bischoff SC, Breda J et al (2020) ESPEN expert statements and practical guidance for nutritional management of individuals with SARS-CoV-2 infection. Clin Nutr 39:16311638. https://doi.org/10.1016/j.clnu.2020.03.022

17. Landi F, Gremese E, Bernabei R et al (2020) Post-COVID-19 global health strategies: the need for an interdisciplinary approach. Aging Clin Exp Res 32:1613-1620. https://doi.org/ 10.1007/s40520-020-01616-x

18. Wu D, Lewis ED, Pae M et al (2019) Nutritional modulation of immune function: analysis of evidence, mechanisms, and clinical relevance. Front Immunol 10:3160. https://doi.org/10.3389/ fimmu.2018.03160

19. Zucchelli A, Bologna E, Marengoni A (2021) Why data on frailty and SARS-CoV-2 infection are basic to progress. Aging Clin Exp Res 33:1429-1432. https://doi.org/10.1007/ s40520-021-01846-7

20. Lidoriki I, Frountzas M, Schizas D (2020) Could nutritional and functional status serve as prognostic factors for COVID-19 in the elderly? Med Hypotheses 144:109946. https://doi.org/10.1016/j. mehy.2020.109946

21. Clegg A, Young J, Iliffe S et al (2013) Frailty in elderly people. Lancet 381:752-762. https://doi.org/10.1016/S0140-6736(12) 62167-9

22. Cruz-Jentoft AJ, Bahat G, Bauer J et al (2019) Sarcopenia: revised European consensus on definition and diagnosis. Age Ageing 48:16-31. https://doi.org/10.1093/ageing/afy169

23. Okazaki T, Ebihara S, Mori T et al (2020) Association between sarcopenia and pneumonia in older people. Geriatr Gerontol Int 20:7-13. https://doi.org/10.1111/ggi.13839

24. Wang P-Y, Li Y, Wang Q (2021) Sarcopenia: an underlying treatment target during the COVID-19 pandemic. Nutrition. https:// doi.org/10.1016/j.nut.2020.111104

25. Leij-Halfwerk S, Verwijs MH, van Houdt S et al (2019) Prevalence of protein-energy malnutrition risk in European older adults in community, residential and hospital settings, according to 22 
malnutrition screening tools validated for use in adults $\geq 65$ years: a systematic review and meta-analysis. Maturitas 126:80-89. https://doi.org/10.1016/j.maturitas.2019.05.006

26. O’Caoimh R, Sezgin D, O’Donovan MR et al (2021) Prevalence of frailty in 62 countries across the world: a systematic review and meta-analysis of population-level studies. Age Ageing 50:96-104. https://doi.org/10.1093/ageing/afaa219

27. Shafiee G, Keshtkar A, Soltani A et al (2017) Prevalence of sarcopenia in the world: a systematic review and meta- analysis of general population studies. J Diabetes Metab Disord. https:// doi.org/10.1186/s40200-017-0302-X

28. Woolford SJ, D'Angelo S, Curtis EM et al (2020) COVID-19 and associations with frailty and multimorbidity: a prospective analysis of UK Biobank participants. Aging Clin Exp Res 32:1897-1905. https://doi.org/10.1007/s40520-020-01653-6

29. Del Brutto OH, Costa AF, Recalde BY et al (2020) Frailty and SARS-CoV-2 infection. A population-based study in a highly endemic village. J Neurol Sci. https://doi.org/10.1016/j.jns. 2020.117136

30. Beaudart C, Reginster JY, Petermans J et al (2015) Quality of life and physical components linked to sarcopenia: the SarcoPhAge study. Exp Gerontol 69:103-110. https://doi.org/10. 1016/j.exger.2015.05.003

31. Balestroni G, Bertolotti G (2015) EuroQol-5D (EQ-5D): an instrument for measuring quality of life. Monaldi Arch Chest Dis 78:155-159. https://doi.org/10.4081/monaldi.2012.121

32. Geerinck $A$, Locquet $M$, Bruyère $O$ et al (2021) Evaluating quality of life in frailty: applicability and clinimetric properties of the SarQoL ${ }^{\circledR}$ questionnaire. J Cachexia Sarcopenia Muscle. https://doi.org/10.1002/jcsm.12687

33. Jenkinson C (1998) The SF-36 physical and mental health summary measures: an example of how to interpret scores. J Heal Serv Res Policy 3:92-96. https://doi.org/10.1177/1355819698 00300206

34. Nestlé Nutrition Institute Nutrition screening as easy as MNA: a guide to complete the Mini Nutiritonal Assessment (MNA). Available online: https://www.mna-elderly.com/forms/mna guide_english.pdf. Accessed on 17 Feb 2021

35. Vellas B, Villars H, Abellan G et al (2006) Overview of the $\mathrm{MNA}^{\circledR}$ - its history and challenges. J Nutr Heal Aging $10: 456-463$

36. Kaiser MJ, Bauer JM, Ramsch C et al (2009) Validation of the mini nutritional assessment short-form $\left(\mathrm{MNA}^{\circledR}-\mathrm{SF}\right)$ : a practical tool for identification of nutritional status. J Nutr Health Aging. https://doi.org/10.1007/s12603-009-0214-7

37. Rubenstein LZ, Harker JO, Salvà A et al (2001) Screening for undernutrition in geriatric practice: developing the short-form mini-nutritional assessment (MNA-SF). J Gerontol 56:366-372. https://doi.org/10.1093/gerona/56.6.M366

38. Corcoran C, Murphy C, Culligan EP et al (2019) Malnutrition in the elderly. Sci Prog 102:171-180. https://doi.org/10.1177/ 0036850419854290

39. Guigoz Y, Vellas B (2021) Nutritional assessment in older adults: MNA ${ }^{\circledR} 25$ years of a screening tool and a reference standard for care and research; what next? J Nutr Health Aging. https://doi.org/10.1007/s12603-021-1601-y

40. Roberts HC, Denison HJ, Martin HJ et al (2011) A review of the measurement of grip strength in clinical and epidemiological studies: towards a standardised approach. Age Ageing 40:423429. https://doi.org/10.1093/ageing/afr051

41. Fried LP, Tangen CM, Walston J et al (2001) Frailty in older adults: evidence for a phenotype. J Gerontol A Biol Med Sci $56: 146-156$

42. Orme JG, Reis J, Herz EJ (1984) Factorial and discriminant validity of the center for epidemiological studies depression (CES-D) scale. J Clin Psychol 42:28-33
43. Taylor HL, Jacobs DR, Schucker B et al (1978) A questionnaire for the assessment of leisure time physical activities. J Chronic Dis 31:741-755. https://doi.org/10.1016/0021-9681(78)90058-9

44. Zadak Z, Hyspler R, Ticha A et al (2013) Polypharmacy and malnutrition. Curr Opin Clin Nutr Metab Care 16:50-55. https://doi.org/10.1097/MCO.0b013e32835b612e

45. Streicher M, van Zwienen-Pot J, Bardon L et al (2018) Determinants of incident malnutrition in community-dwelling older adults: a Manuel multicohort meta-analysis. J Am Geriatr Soc 66:2335-2343. https://doi.org/10.1111/jgs.15553

46. Bokhorst-de V, van der Schueren MAE, Lonterman-Monasch $S$ et al (2013) Prevalence and determinants for malnutrition in geriatric outpatients. Clin Nutr. https://doi.org/10.1016/j.clnu. 2013.05.007

47. Coelho T, Paúl C, Gobbens RJJ et al (2015) Determinants of frailty: The added value of assessing medication. Front Aging Neurosci. https://doi.org/10.3389/fnagi.2015.00056

48. Tombaugh TN, McIntyre NJ (1992) The Mini-mental state examination: a comprehensive review. J Am Geriatr Soc 40:922-935. https://doi.org/10.1111/j.1532-5415.1992.tb019 92. $\mathrm{x}$

49. Abate SM, Chekole YA, Estifanos MB et al (2021) Prevalence and outcomes of malnutrition among hospitalized COVID-19 patients: a systematic review and meta-analysis. Clin Nutr ESPEN 43:174-183. https://doi.org/10.1016/j.clnesp.2021.03.002

50. Wierdsma NJ, Kruizenga HM, Konings LA et al (2021) Poor nutritional status, risk of sarcopenia and nutrition related complaints are prevalent in COVID-19 patients during and after hospital admission. Clin Nutr ESPEN 43:369-376. https://doi.org/10. 1016/j.clnesp.2021.03.021

51. Morley JE, Kalantar-Zadeh K, Anker SD (2020) COVID-19: a major cause of cachexia and sarcopenia? J Cachexia Sarcopenia Muscle 11:863-865. https://doi.org/10.1002/jcsm.12589

52. Li T, Zhang Y, Gong C et al (2020) Prevalence of malnutrition and analysis of related factors in elderly patients with COVID-19 in Wuhan, China. Eur J Clin Nutr 74:871-875. https://doi.org/10. 1038/s41430-020-0642-3

53. Yu Y, Ye J, Chen M et al (2021) Erratum to: malnutrition prolongs the hospitalization of patients with COVID-19 infection: a clinical epidemiological analysis (The journal of nutrition, health and aging, (2020), 10.1007/s12603-020-1541-y). J Nutr Heal Aging 25:369-373

54. Mertens E, Peñalvo JL (2021) The burden of malnutrition and fatal COVID-19: a global burden of disease analysis. Front Nutr 7:1-12. https://doi.org/10.3389/fnut.2020.619850

55. Kim J-W, Yoon JS, Kim EJ et al (2021) Prognostic implication of baseline sarcopenia for length of hospital stay and survival in patients with coronavirus disease 2019. J Gerontol Ser A. https:// doi.org/10.1093/gerona/glab085

56. Hanlon P, Nicholl BI, Jani BD et al (2018) Frailty and pre-frailty in middle-aged and older adults and its association with multimorbidity and mortality: a prospective analysis of 493737 UK Biobank participants. Lancet Public Health 3:e323-e332. https:// doi.org/10.1016/S2468-2667(18)30091-4

57. Wyper GMA, Assunção R, Cuschieri S et al (2020) Population vulnerability to COVID-19 in Europe: a burden of disease analysis. Arch Public Heal 78:1-8. https://doi.org/10.1186/ s13690-020-00433-y

58. Bustos Sierra N, Bossuyt N, Braeye T et al (2020) All-cause mortality supports the COVID-19 mortality in Belgium and comparison with major fatal events of the last century. Arch Public Heal 78:1-8. https://doi.org/10.1186/s13690-020-00496-x

59. Dumitrascu F, Branje KE, Hladkowicz ES et al (2021) Association of frailty with outcomes in individuals with COVID-19: a living review and meta-analysis. J Am Geriatr Soc. https://doi.org/10. 1111/jgs.17299 
60. Cederholm T (2015) Overlaps between frailty and sarcopenia definitions. Nestle Nutr Inst Workshop Ser 83:65-69. https://doi.org/ $10.1159 / 000382063$

61. Nascimento CM, Ingles M, Salvador-Pascual A et al (2019) Sarcopenia, frailty and their prevention by exercise. Free Radic Biol Med 132:42-49. https://doi.org/10.1016/j.freeradbiomed.2018.08. 035

62. Sofra X, Badami S (2020) Adverse effects of sedentary lifestyles: inflammation, and high-glucose induced oxidative stress-a double blind randomized clinical trial on diabetic and prediabetic patients. Health (Irvine Calif) 12:1029-1048. https://doi.org/10. 4236/health.2020.128076

63. Nilsson A, Bergens O, Kadi F (2018) Physical activity alters inflammation in older adults by different intensity levels. Med Sci Sports Exerc 50:1502-1507. https://doi.org/10.1249/MSS. 0000000000001582

64. da Silveira MP, da Silva Fagundes KK, Bizuti MR et al (2021) Physical exercise as a tool to help the immune system against COVID-19: an integrative review of the current literature. Clin Exp Med 21:15-28. https://doi.org/10.1007/s10238-020-00650-3

65. Harvey JA, Chastin SFM, Skelton DA (2013) Prevalence of sedentary behavior in older adults: a systematic review. Int J Environ Res Public Health 10:6645-6661. https://doi.org/10.3390/ijerp h10126645
66. Negm AM, Kennedy CC, Thabane L et al (2019) Management of frailty: a systematic review and network meta-analysis of randomized controlled trials. J Am Med Dir Assoc 20:1190-1198. https://doi.org/10.1016/j.jamda.2019.08.009

67. Antonelli M, Penfold RS, Merino J et al (2021) Risk factors and disease profile of post-vaccination SARS-CoV-2 infection in UK users of the COVID Symptom Study app: a prospective, community-based, nested, case-control study. Lancet Infect Dis. https:// doi.org/10.1016/S1473-3099(21)00460-6

68. Folsom AR, Jacobs DR, Caspersen CJ et al (1986) Test-retest reliability of the Minnesota leisure time physical activity questionnaire. J Chronic Dis 39:505-511. https://doi.org/10.1016/00219681(86)90195-5

69. Richardson MT, Leon AS, Jacobs DR et al (1994) Comprehensive evaluation of the Minnesota leisure time physical activity questionnaire. J Clin Epidemiol 47:271-281. https://doi.org/10.1016/ 0895-4356(94)90008-6

Publisher's Note Springer Nature remains neutral with regard to jurisdictional claims in published maps and institutional affiliations. 